\title{
Monitoring the temperature of a direct contact membrane distillation.
}

\author{
Amin Boumenir $^{1}$ | Mohamed Ghattassi ${ }^{2}$ | Taous-Meriem Laleg ${ }^{2}$
}

\author{
${ }^{1}$ Department of Mathematics, University of \\ West Georgia, Carrollton, GA 30118, \\ United States. \\ Email: boumenir@westga.edu \\ ${ }^{2}$ Computer, Electrical, and Mathematical \\ Sciences and Engineering Division, King \\ Abdullah University of Science and \\ Technology (KAUST), Thuwal, \\ 53955-6900, Kingdom of Saudi Arabia. \\ Email: mohamed.ghattassi@kaust.edu.sa \\ Email: taousmeriem.laleg@kaust.edu.sa
}

\begin{abstract}
Summary
This paper deals with the heat transfer monitoring occurring within an inaccessible membrane distillation system. The membrane separates heated sea water and filtered cooled drinkable water. By adjusting the temperature of the incoming heated sea water and knowing its temperature distribution, engineers can keep its temperature within its best operating parameters and avoid hot spots to form. This would help prolong its life cycle and minimize the cost of the distillation process. In particular, we show that an external observation is enough to reconstruct the temperature of the membrane which is considered as an unknown source term in a parabolic system.
\end{abstract}

\section{KEYWORDS:}

Parameters Identification, Inverse parabolic system, Direct Contact Membrane Distillation System.

\section{1 | INTRODUCTION}

The livelihood of people living in arid regions, but with access to sea or salty water depends on automated and efficient desalination processes. Recent droughts and rising temperatures due to global warming make the development of desalination technologies crucial and a priority to many parts of the world. However, conventional desalination systems are costly and energy inefficient. Therefore there is a tremendous effort to develop new environmentally friendly desalination technologies with affordable operational cost. Among the emerging desalination technologies is the membrane distillation and in particular the Direct Contact Membrane Distillation (DCMD), with its simplicity and low running cost ${ }^{1}$. The DCMD consists of two large containers; The first one runs hot seawater while the second one contains drinkable cold water and both are separated by a hydrophobic membrane. It can achieve low cost by recycling the exhaust heat and also by using low grade renewable thermal energies, such as solar power, to heat up the sea water. The essence of the process is based on the atomic structure of the hydrophobic membrane and the temperature gradient between the two sides of the membrane, which allows vapor to pierce through it, while blocking the liquid phase. As steam permeates through the membrane, it also condenses by exchanging heat transfer which can easily build up. Moreover, DCMD has a big advantage of operating at low temperatures and pressure comparing to conventional desalination processes. However, it has a lower production rate and is subject to fouling which consists of salt deposits on the surface of the membrane blocking the vapor transfer. Thus, to allow for a better operation of the DCMD system, it is crucial to constantly monitor the temperature at the membrane surface in real time, see 2 .

Many studies have been conducted recently by engineers to propose accurate mathematical models of MD systems ${ }^{4 / 56677 .}$ For example, a system of two dimensional parabolic equations coupled at the boundary has been recently proposed for the DCMD configuration and has been validated experimentally in $\frac{466}{6}$. The two parabolic equations model the heat transfer in the two tanks and the mass transfer through the membrane provides a coupling equation at the membrane interface. However the temperature at the membrane surface is unknown and inaccessible for measurements and thus needs to be estimated from boundary observations. 
There is a growing interest in inverse problem problems dealing with the reconstruction of physical parameters in systems of partial differential equations (PDEs), recovery of a single distributed conductance parameter on a finite interval and a graph. Moreover, an extension of $\frac{17}{17}$ is given in $\frac{16}{16}$ where the authors show how unknown coefficients and source terms for a parabolic equation can be recovered from the dynamical Neumann-to-Dirichlet map associated with the boundary vertices. From observation on the infinite interval of time and in a bounded open, connected domain in $\mathbb{R}^{d}, d \geqslant 2$, Al-Musallam and Boumenir ${ }^{18}$ also considered an inverse problem of recovering a coefficient and source term in a parabolic problem. More recently, in ${ }^{15}$, Boumenir presents two methods depending on the spectrum of the system for the problem of reconstruction of the coefficients of a linear parabolic system from finite time observations of the solution on the boundary. There is also a large literature on stability estimates for the inverse problem

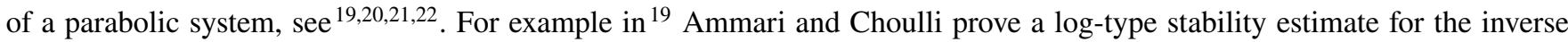
problem consisting in determining both the potential and the damping coefficient in a dissipative wave equation from boundary measurements. Then, they show how we can adapt their approach to the heat equation. Their proofs are based on observability inequalities for exactly controllable systems and spectral decompositions. More recently Ammari, Choulli and Triki ${ }^{23}$ improve the result presented in $\frac{19}{19}$ where they obtained a local Hölder stability instead of a logarithmic stability.

The goal of this work is to recover the temperature on the surface of the inaccessible membrane of the DCMD parabolic system by boundary observations. To circumvent the missing boundary data on the membrane, we use external observations of the system and turn the problem into a Cauchy problem in the $x$-direction. Thus the initial coupled non-homogeneous boundary value problems turn into a decoupled boundary conditions but with new source terms. In other words, the heated sea water is seen as a heat source for the distilled water side, and vice-versa. This leads to a simple representation of the solution that allows us to identify the source terms, which would reveal the temperature on both sides of the membrane. To use Fourier type methods, we first recast the system into a self-adjoint form so we can integrate the solution and can easily identify the temperature of the membrane.

The paper is organized as follows. In the next section, the mathematical model of the heat transfer in DCMD is presented with a co-current configuration, that is both sea water and drinkable water flow in the same direction. Section 3 is devoted to the changes of variables, which help transform the boundary coupling into a source term and also recast the system into a selfadjoint problem. In addition, we prove that the performed change of variables is a bijection. In section 4 the source term is recovered using a series representation in terms of basis functions. Then by reading part of the boundary and using the Laplace transform, the temperature on the membrane's surface can be recovered.

\section{2 | MATHEMATICAL MODEL OF A DIRECT CONTACT MEMBRANE DISTILLATION SYSTEM}

Denote by $f(t, x, y)$ the temperature of the incoming heated sea water and by $p(t, x, y)$ the temperature of the outgoing cold filtered water at time $t$ and at the point $(x, y)$ in $\left[0, \ell_{1}\right] \times\left[0, \ell_{2}\right]$ and $\left[\delta_{m}+\ell_{1}, \delta_{m}+2 \ell_{1}\right] \times\left[0, \ell_{2}\right]$ respectively, (here $\delta_{m}$ denotes thickness of the membrane, see Fig. 11). Denote by the sea water domain by $\Omega_{1}=\left(0, \ell_{1}\right) \times\left(0, \ell_{2}\right)$ and the filtered water domain $\Omega_{2}=\left(\delta_{m}+\ell_{1}, \delta_{m}+2 \ell_{1}\right) \times\left(0, \ell_{2}\right)$. Thus the functions $f(t, x, y)$ and $p(t, x, y)$ are solutions of

$$
\begin{cases}\partial_{t} f(t, x, y)-\alpha_{f} \Delta f(t, x, y)+\beta_{f} \partial_{y} f(t, x, y)=0 & t>0,(x, y) \in \Omega_{1}, \\ \partial_{t} p(t, x, y)-\alpha_{p} \Delta p(t, x, y)+\beta_{p} \partial_{y} p(t, x, y)=0 & t>0,(x, y) \in \Omega_{2}, \\ \partial_{x} f\left(t, \ell_{1}, y\right)=\gamma_{f}\left(f\left(t, \ell_{1}, y\right)-p\left(t, \ell_{1}+\delta_{m}, y\right)\right) & t>0, y \in\left(0, \ell_{2}\right), \\ \partial_{x} p\left(t, \ell_{1}+\delta_{m}, y\right)=\gamma_{p}\left(f\left(t, \ell_{1}, y\right)-p\left(t, \ell_{1}+\delta_{m}, y\right)\right) & t>0, y \in\left(0, \ell_{2}\right), \\ \partial_{x} f(t, 0, y)=0 & t>0, y \in\left(0, \ell_{2}\right), \\ \partial_{x} p\left(t, \delta_{m}+2 \ell_{1}, y\right)=0 & t>0, y \in\left(0, \ell_{2}\right), \\ p(t, x, 0)=T_{p}(t, x) & t>0, x \in\left(\delta_{m}+\ell_{1}, \delta_{m}+2 \ell_{1}\right), \\ \partial_{y} p\left(t, x, \ell_{2}\right)=0 & t>0, x \in\left(\delta_{m}+\ell_{1}, \delta_{m}+2 \ell_{1}\right), \\ f(t, x, 0)=T_{f}(t, x) & t>0, x \in\left(0, \ell_{1}\right), \\ \partial_{y} f\left(t, x, \ell_{2}\right)=0 & t>0, x \in\left(0, \ell_{1}\right), \\ f(0, x, y)=f_{0}(x, y) & (x, y) \in \Omega_{1}, \\ p(0, x, y)=p_{0}(x, y) & (x, y) \in \Omega_{2} .\end{cases}
$$


The coefficients $\alpha_{f}$ and $\alpha_{p}$ are assumed to be given positive constants, that depend on the thermal conductivity and on the

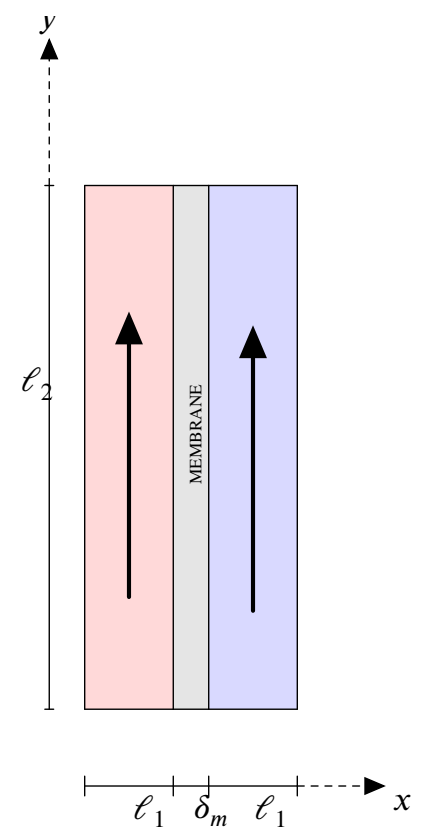

FIGURE 1 Schematic of the DCMD system

densities of the fluids ( $\sec ^{7}$ )

$$
\alpha_{f}=\frac{\kappa_{f}}{\rho_{f} C_{f}}, \text { and } \alpha_{p}=\frac{\kappa_{p}}{\rho_{p} C_{p}},
$$

where $\kappa_{k}, \rho_{k}$ and $C_{k}$, for $k=p, f$ denote respectively, the thermal conductivity, liquid density and specific heat capacity of the fluid. The constants $\gamma_{f}$ and $\gamma_{p}$ are positive and control the heat transfer through the boundary, see ${ }^{7}$. The coefficients $\beta_{f}>0$ and $\beta_{p}>0$ denote the velocities of the flow in the feed and permeate side, respectively.

Our objective is to recover the temperature on both sides of the membrane, that is $f\left(t, \ell_{1}, y\right)$ and $p\left(t, \ell_{1}+\delta_{m}, y\right)$, by external boundary measurements on the accessible side $x=0$ and $0 \leq y \leq \ell_{2}$.

\section{3 | CHANGES OF VARIABLES}

\section{1 | Common Domain}

The first step is to use the same domain for both functions $f$ and $p$. To this end we map the domain $\Omega_{2}$ onto $\Omega_{1}$, by reflection and translation, such that the side $x=2 \ell_{1}+\delta_{m}$ is mapped onto $x=0$ and $x=\ell_{1}+\delta_{m}$ onto $\ell_{1}$. Thus we rename both as $\Omega$, i.e. $\Omega=-\Omega_{2}+2 \ell_{1}+\delta_{m}=\Omega_{1}$. Clearly $f$ is invariant under the change of variables, as $\Omega=\Omega_{1}$ while the new variable temperature $p_{\text {new }}$ satisfies

$$
p_{\text {new }}(t, x, y)=p\left(t, 2 \ell_{1}+\delta_{m}-x, y\right) \quad t \geqslant 0, \forall(x, y) \in \Omega
$$

For the sake of simplicity we still denote by $p$ the new variable $p_{\text {new }}$. To quickly identify the sides of $\Omega$, let us denote the bottom side by $\Gamma_{1}:=\left(0, \ell_{1}\right) \times\{0\}$, the left side by $\Gamma_{2}:=\{0\} \times\left(0, \ell_{2}\right)$, the top one by $\Gamma_{3}:=\left(0, \ell_{1}\right) \times\left\{\ell_{2}\right\}$, and finally the right side by $\Gamma_{4}:=\left\{\ell_{1}\right\} \times\left(0, \ell_{2}\right)$. 


\section{2 | Decoupling the boundary condition}

We first decouple the boundary conditions on $x=\ell_{1}$ in 2.1 by introducing the new variables

$$
\begin{aligned}
& \tilde{f}(t, x, y)=f(t, x, y)-p\left(t, \ell_{1}, y\right), \quad t>0,(x, y) \in \bar{\Omega} \\
& \tilde{p}(t, x, y)=p(t, x, y)-f\left(t, \ell_{1}, y\right), \quad t>0,(x, y) \in \bar{\Omega} .
\end{aligned}
$$

to obtain

$$
\left\{\begin{array}{lc}
\partial_{t} \tilde{f}(t, x, y)-\alpha_{f} \Delta \tilde{f}(t, x, y)+\beta_{f} \partial_{y} \tilde{f}(t, x, y)=G_{1}(t, y), & t>0,(x, y) \in \Omega, \\
\partial_{x} \tilde{f}\left(t, \ell_{1}, y\right)=-\gamma_{f} \tilde{f}\left(t, \ell_{1}, y\right) & t>0,(x, y) \in \Gamma_{4}, \\
\partial_{x} \tilde{f}(t, 0, y)=0 & t>0,(x, y) \in \Gamma_{2}, \\
\tilde{f}(t, x, 0)=T_{\tilde{f}}(t, x) & t>0,(x, y) \in \Gamma_{1}, \\
\partial_{y} \tilde{f}\left(t, x, \ell_{2}\right)=0 & t>0,(x, y) \in \Gamma_{3}, \\
\tilde{f}(0, x, y)=\tilde{f}_{0}(x, y) & (x, y) \in \Omega,
\end{array}\right.
$$

and

$$
\left\{\begin{array}{lc}
\partial_{t} \tilde{p}(t, x, y)-\alpha_{p} \Delta \tilde{p}(t, x, y)+\beta_{p} \partial_{y} \tilde{p}(t, x, y)=G_{2}(t, y), & t>0,(x, y) \in \Omega, \\
\partial_{x} \tilde{p}\left(t, \ell_{1}, y\right)=-\gamma_{p} \tilde{p}\left(t, \ell_{1}, y\right) & t>0,(x, y) \in \Gamma_{4}, \\
\partial_{x} \tilde{p}(t, 0, y)=0 & t>0,(x, y) \in \Gamma_{2}, \\
\tilde{p}(t, x, 0)=T_{\tilde{p}}(t, x) & t>0,(x, y) \in \Gamma_{1}, \\
\partial_{y} \tilde{p}\left(t, x, \ell_{2}\right)=0 & t>0,(x, y) \in \Gamma_{3}, \\
\tilde{p}(0, x, y)=\tilde{p}_{0}(x, y) & (x, y) \in \Omega,
\end{array}\right.
$$

where the new source terms are

$$
\begin{aligned}
& G_{1}(t, y)=-\partial_{t} p\left(t, \ell_{1}, y\right)+\alpha_{f} \partial_{y y} p\left(t, \ell_{1}, y\right)-\beta_{f} \partial_{y} p\left(t, \ell_{1}, y\right), \quad t>0(x, y) \in \Gamma_{4}, \\
& G_{2}(t, y)=-\partial_{t} f\left(t, \ell_{1}, y\right)+\alpha_{p} \partial_{y y} f\left(t, \ell_{1}, y\right)-\beta_{p} \partial_{y} f\left(t, \ell_{1}, y\right), \quad t>0(x, y) \in \Gamma_{4},
\end{aligned}
$$

and also where for $x \in\left(0, \ell_{1}\right)$ and $t>0$

$$
\begin{array}{rr}
T_{\tilde{f}}(t, x)=T_{f}(t, x)-T_{p}\left(t, \ell_{1}\right) & T_{\tilde{p}}(t, x)=T_{p}(t, x)-T_{f}\left(t, \ell_{1}\right) \\
\tilde{f}_{0}(x, y)=f_{0}(x, y)-p_{0}\left(\ell_{1}, y\right), & \tilde{p}_{0}(x, y)=p_{0}(x, y)-f_{0}\left(\ell_{1}, y\right) .
\end{array}
$$

Obviously (3.7) are the new boundary conditions for (3.3) and (3.4) which are obtained from the boundary conditions in $(2.1)$.

\section{3 | Self-Adjoint form}

We now rescale the solution to turn (3.3) and (3.4) into self-adjoint form

$$
\begin{array}{ll}
g(t, x, y)=\tilde{f}(t, x, y) e^{-\frac{\beta_{f}}{2 \alpha_{f}} y}, \quad t \geq 0, \quad(x, y) \in \Omega, \\
q(t, x, y)=\tilde{p}(t, x, y) e^{-\frac{\beta_{p}}{2 \alpha_{p}} y}, \quad t \geq 0, \quad(x, y) \in \Omega,
\end{array}
$$

so we can use the Fourier series. Under the transformation (3.9), system 3.3 becomes

$$
\begin{cases}\partial_{t} g(t, x, y)-\alpha_{f} \Delta g(t, x, y)+\frac{\beta_{f}^{2}}{4 \alpha_{f}} g(t, x, y)=F_{1}(t, y) & t>0,(x, y) \in \Omega, \\ \partial_{x} g\left(t, \ell_{1}, y\right)=-\gamma_{f} g\left(t, \ell_{1}, y\right) & t>0,(x, y) \in \Gamma_{4}, \\ \partial_{x} g(t, x, y)=0 & t>0,(x, y) \in \Gamma_{2}, \\ g(t, x, 0)=T_{\tilde{f}}(t, x) & t>0,(x, y) \in \Gamma_{1}, \\ \partial_{y} g\left(t, x, \ell_{2}\right)=-\frac{\beta_{f}}{2 \alpha_{f}} g\left(t, x, \ell_{2}\right) & t>0,(x, y) \in \Gamma_{3}, \\ g(0, x, y)=g_{0}(x, y) & t>0,(x, y) \in \Omega,\end{cases}
$$


and

$$
\left\{\begin{array}{lc}
\partial_{t} q(t, x, y)-\alpha_{p} \Delta q(t, x, y)+\frac{\beta_{p}^{2}}{4 \alpha_{p}} q(t, x, y)=F_{2}(t, y) & t>0,(x, y) \in \Omega, \\
\partial_{x} q(t, x, y)=-\gamma_{p} q(t, x, y) & t>0,(x, y) \in \Gamma_{4}, \\
\partial_{x} q(t, x, y)=0 & t>0,(x, y) \in \Gamma_{2}, \\
q(t, x, y)=T_{\tilde{p}}(t, x) & t>0,(x, y) \in \Gamma_{1}, \\
\partial_{y} q(t, x, y)=-\frac{\beta_{p}}{2 \alpha_{p}} q(t, x, y) & t>0,(x, y) \in \Gamma_{3}, \\
q(0, x, y)=q_{0}(x, y) & (x, y) \in \Omega,
\end{array}\right.
$$

where the new sources $F_{1}, F_{2}$ are now given by

$$
F_{1}(t, y)=G_{1}(t, y) e^{-\frac{\beta_{f}}{2 \alpha_{f}} y}, \text { and } F_{2}(t, y)=G_{2}(t, y) e^{-\frac{\beta_{p}}{2 \alpha_{p}} y} .
$$

As $F_{1}$ depends on $G_{1}, 3.12$, which in turns depends on $p$, see (3.5) and so $F_{1}$ is an unknown source to be determined. Observe that we have symmetric boundary conditions on $\Gamma_{2}, \Gamma_{3}$ and $\Gamma_{4}$. Moreover, the value of the solution $(g, q)$ on $\Gamma_{1}$ is given. To proceed further we need to see that the change of variables allows us to go back to the original variables $f$ and $p$.

\section{4 | The change of variables}

We now prove the invertibility of the change of variables $3.1-(3.2)$, namely

$$
\begin{aligned}
& \tilde{f}(t, x, y)=f(t, x, y)-p\left(t, \ell_{1}, y\right), \quad t>0,(x, y) \in \bar{\Omega} \\
& \tilde{p}(t, x, y)=p(t, x, y)-f\left(t, \ell_{1}, y\right), \quad t>0,(x, y) \in \bar{\Omega} .
\end{aligned}
$$

It is obvious that a given pair $(f, p)$ in $\bar{\Omega}$, will uniquely determine $(\tilde{f}, \tilde{p})$ there. Conversely, once we have obtained $\tilde{f}(t, x, y)$ and $\tilde{p}(t, x, y)$, can we still get uniquely the original solution $f(t, x, y)$ and $p(t, x, y)$ ? This inverse map is not so obvious as it requires the prior knowledge of the functions $p\left(t, \ell_{1}, y\right)$ and $f\left(t, \ell_{1}, y\right)$ first.

To this end we need to show that they can be reconstructed from $(\tilde{f}, \tilde{p})$. Given the latter, we know $(g, q)$, by $\sqrt{3.9}$, the solutions of (3.10)-3.11), and thus we know the source terms $\left(F_{1}, F_{2}\right)$ and equivalently $G_{1}$ and $G_{2}$ in 3.12$)$. Observe that the temperature at the membrane, $f\left(t, \ell_{1}, y\right)$ and $p\left(t, \ell_{1}, y\right)$ are respectively the solutions of the following simple one-dimensional parabolic equation, which are given in (3.5) and (3.6)

$$
\left\{\begin{array}{lc}
\partial_{t} f\left(t, \ell_{1}, y\right)-\alpha_{p} \partial_{y y} f\left(t, \ell_{1}, y\right)+\beta_{p} \partial_{y} f\left(t, \ell_{1}, y\right)=-G_{2}(t, y) & t>0, y \in\left(0, \ell_{2}\right), \\
f\left(t, \ell_{1}, 0\right)=T_{f}\left(t, \ell_{1}\right) & t>0, \\
\partial_{y} f\left(t, \ell_{1}, \ell_{2}\right)=0 & t>0, \\
f\left(0, \ell_{1}, y\right)=f_{0}\left(\ell_{1}, y\right) & \left(0, \ell_{2}\right),
\end{array}\right.
$$

and

$$
\begin{cases}\partial_{t} p\left(t, \ell_{1}, y\right)-\alpha_{f} \partial_{y y} p\left(t, \ell_{1}, y\right)+\beta_{f} \partial_{y} p\left(t, \ell_{1}, y\right)=-G_{1}(t, y) & t>0, y \in\left(0, \ell_{2}\right), \\ p\left(t, \ell_{1}, 0\right)=T\left(t, \ell_{1}\right) & t>0, \\ \partial_{y} p\left(t, \ell_{1}, \ell_{2}\right)=0 & t>0, \\ p\left(0, \ell_{1}, y\right)=p_{0}\left(\ell_{1}, y\right) & \left(0, \ell_{2}\right) .\end{cases}
$$

Obviously to find explicitly $f\left(t, \ell_{1}, y\right)$ we only need to know the boundary conditions for $t>0$ and $y \in\left(0, \ell_{2}\right)$

$$
f\left(t, \ell_{1}, 0\right)=T_{f}\left(t, \ell_{1}\right), \quad \partial_{y} f\left(t, \ell_{1}, \ell_{2}\right)=0 ; \quad f\left(0, \ell_{1}, y\right)=f_{0}\left(\ell_{1}, y\right)
$$

which are already given in 2.1 . Similarly we can find $p\left(t, \ell_{1}, y\right)$ which would yield $p(t, x, y)$ and consequently we have proved that

Proposition 1. The change of variables defined by (3.1)-(3.2),

$$
\begin{aligned}
& f(t, x, y)=\tilde{f}(t, x, y)+p\left(t, \ell_{1}, y\right), \quad \text { for } \quad t>0,(x, y) \in \bar{\Omega} \\
& p(t, x, y)=\tilde{p}(t, x, y)+f\left(t, \ell_{1}, y\right), \quad \text { for } \quad t>0,(x, y) \in \bar{\Omega} .
\end{aligned}
$$

is a bijection. 


\section{4 | RECONSTRUCTING THE TEMPERATURE FROM THE BOUNDARY BY READING ON $\Gamma_{2}$}

We shall need a few preliminary steps to work out the direct problem before we move to the inverse problem. First observe that the normalized eigenfunctions for the operator appearing in 3.10 )

$$
\begin{cases}-\alpha_{f} \Delta \varphi_{n k}(x, y)+\frac{\beta_{f}^{2}}{4 \alpha_{f}} \varphi_{n k}(x, y)=\lambda_{n k} \varphi_{n k} & (x, y) \in \Omega, \\ \partial_{x} \varphi_{n k}\left(\ell_{1}, y\right)=-\gamma_{f} \varphi_{n k}\left(\ell_{1}, y\right) & (x, y) \in \Gamma_{4} \\ \partial_{x} \varphi_{n k}(0, y)=0 & (x, y) \in \Gamma_{2}, \\ \varphi_{n k}(x, y)=0 & (x, 0) \in \Gamma_{1}, \\ \partial_{y} \varphi_{n k}\left(x, \ell_{2}\right)=-\frac{\beta_{f}}{2 \alpha_{f}} \varphi_{n k}\left(x, \ell_{2}\right) & (x, y) \in \Gamma_{3} .\end{cases}
$$

are explicitly given by

$$
\varphi_{n k}(x, y)=r_{n} s_{k} \cos \left(x \mu_{n}\right) \sin \left(y v_{k}\right)
$$

with

$$
r_{n}=\left(\int_{0}^{\ell_{1}} \cos ^{2}\left(x \mu_{n}\right) d x\right)^{-1 / 2} \quad \text { and } \quad s_{k}=\left(\int_{0}^{\ell_{2}} \sin ^{2}\left(y v_{k}\right) d y\right)^{-1 / 2}
$$

and $\left\{\mu_{n}\right\}_{n \geq 1}$ and $\left\{v_{k}\right\}_{k \geq 1}$ are the positive roots of

$$
-\mu_{n} \sin \left(\ell_{1} \mu_{n}\right)+\gamma_{f} \cos \left(\ell_{1} \mu_{n}\right)=0, \quad \text { and } \quad v_{k} \cos \left(\ell_{2} v_{k}\right)+\frac{\beta_{f}}{2 \alpha_{f}} \sin \left(\ell_{2} v_{k}\right)=0
$$

with asymptotics

The eigenvalues of (4.1) are then explicitly given by

$$
\mu_{n}=\frac{\pi}{\ell_{1}} n+o(1) \quad \text { and } \quad v_{k}=\frac{\pi}{\ell_{2}}\left(k+\frac{1}{2}\right)+o(1) .
$$

$$
\lambda_{n k}=\alpha_{f}\left(\mu_{n}^{2}+v_{k}^{2}\right)+\frac{\beta_{f}^{2}}{4 \alpha_{f}} \quad \text { for } n, k \geq 1 .
$$

Use the eigen-basis $\left\{\varphi_{n k}\right\}_{n, k \geqslant 1}$, to define the Fourier coefficients of $g_{0} \in L^{2}(\Omega)$ by

$$
c_{n k}\left(g_{0}\right)=\int_{\Omega} g_{0}(x, y) \varphi_{n k}(x, y) d x d y .
$$

Theorem 1. For $g_{0} \in L^{2}(\Omega)$ we can recover the source $F_{1}$ in $(3.10)$ and $p\left(t, \ell_{1}, y\right)$ which is the temperature of the membrane, from the boundary reading of $g(t, 0, y)$ on $\Gamma_{2}$, for $t>0$.

Proof. The proof is made up of few steps. We start off by using the eigenfunctions $\varphi_{n k}$, (4.2), to represent the solution $g$ of (3.11)

$$
g(t, x, y)=\sum_{n, k \geq 1} c_{n k}(t) \varphi_{n k}(x, y) \quad \forall t>0,(x, y) \in \Omega,
$$

where

$$
c_{n k}(t)=\int_{0}^{\ell_{2}} \int_{0}^{\ell_{1}} g(t, x, y) \varphi_{n k}(x, y) d x d y .
$$

For $t>0$, we know that $g(t, .,.) \in C^{2}(\Omega)$, since it is a solution of a parabolic equation, and so the Fourier series converges uniformly, and

$$
\begin{aligned}
c_{n k}^{\prime}(t) & =\int_{0}^{\ell_{2}} \int_{0}^{\ell_{1}} \alpha_{f} \Delta g(t, x, y) \varphi_{n k}(x, y) d x d y-\frac{\beta_{f}^{2}}{4 \alpha_{f}} c_{n k}(t)+\int_{0}^{\ell_{2}} \int_{0}^{\ell_{1}} F_{1}(t, y) \varphi_{n k}(x, y) d x d y \\
& =b_{n k}(t)-\lambda_{n k} c_{n k}(t)+\int_{0}^{\ell_{2}} \int_{0}^{\ell_{1}} F_{1}(t, y) \varphi_{n k}(x, y) d x d y
\end{aligned}
$$


where $b_{n k}$ is the contour integral over the boundary obtained by integration by parts in Green's formula,

$$
\begin{aligned}
b_{n k}(t) & =\alpha_{f} \sum_{i=1}^{i=4} \int_{\Gamma_{i}} \partial_{n} g(t, x, y) \varphi_{n k}(x, y)-g(t, x, y) \partial_{n} \varphi_{n k}(x, y) d \sigma_{i} \\
& =\alpha_{f} \int_{0}^{\ell_{1}} T_{\tilde{f}}(t, x) \partial_{y} \varphi_{n k}(x, 0) d x=\alpha_{f} s_{k} v_{k} r_{n} \int_{0}^{\ell_{1}} T_{\tilde{f}}(t, x) \cos \left(\mu_{n} x\right) d x \\
& =\alpha_{f} s_{k} v_{k} \theta_{n}(t)
\end{aligned}
$$

which reduces to the boundary term on $\Gamma_{1}$ only and where $\theta_{n}$ are the Fourier coefficients of $T_{\tilde{f}}(t, x)$

$$
\theta_{n}(t)=r_{n} \int_{0}^{\ell_{1}} T_{\tilde{f}}(t, x) \cos \left(\mu_{n} x\right) d x
$$

The remaining integral term is explicitly given by

$$
\int_{0}^{\ell_{2}} \int_{0}^{\ell_{1}} F_{1}(t, y) \varphi_{n k}(x, y) d x d y=r_{n} a_{n} s_{k} \int_{0}^{\ell_{2}} F_{1}(t, y) \sin \left(y v_{k}\right) d y=r_{n} a_{n} \rho_{k}(t),
$$

with

$$
a_{n}=\int_{0}^{\ell_{1}} \cos \left(x \mu_{n}\right) d x \text { and } \rho_{k}(t):=s_{k} \int_{0}^{\ell_{2}} F_{1}(t, y) \sin \left(y v_{k}\right) d y .
$$

The solution to the $c_{n k}$ equation in 4.7 is then given by

$$
c_{n k}(t)=c_{n k}(0) e^{-\lambda_{n k} t}+\alpha_{f} s_{k} \nu_{k} \int_{0}^{t} e^{-\lambda_{n k}(t-\eta)} \beta_{k}(\eta) d \eta+r_{n} a_{n} \int_{0}^{t} e^{-\lambda_{n k}(t-\eta)} \rho_{k}(\eta) d \eta
$$

and denote by

$$
d_{n k}(t)=c_{n k}(0) e^{-\lambda_{n k} t}+\alpha_{f} s_{k} v_{k} \int_{0}^{t} e^{-\lambda_{n k}(t-\eta)} \beta_{k}(\eta) d \eta
$$

to write

$$
c_{n k}(t)=d_{n k}(t)+r_{n} a_{n} \int_{0}^{t} e^{-\lambda_{n k}(t-\eta)} \rho_{k}(\eta) d \eta .
$$

To reconstruct the source term $F_{1}$, we need to find its Fourier coefficients $\rho_{k}(t)$, see (4.9)

$$
F_{1}(t, y)=\sum_{k \geq 1} \rho_{k}(t) s_{k} \sin \left(y v_{k}\right)
$$

by solving the integral equation (4.12). To do so we need to use a boundary observation of the solution $g(t, 0, y)$ on the boundary $\Gamma_{2}$, in order to obtain $c_{n, k}$ that are required in (4.12). However it would reveal the sum of $c_{n k}$ over $n$, while (4.12) makes use of individual $c_{n k}$.

\section{Identifying $\rho_{k}$ from the observation of the solution on $\Gamma_{2}$.}

The observation $g(t, 0, y)$ on $\Gamma_{2}$ is also given by 4.6,

$$
g(t, 0, y)=\sum_{n, k \geq 1} c_{n k}(t) \varphi_{n k}(0, y)=\sum_{n, k \geq 1} c_{n k}(t) r_{n} s_{k} \sin \left(y v_{k}\right) \quad \forall t>0, y \in\left(0, \ell_{2}\right) .
$$

Note that since the observation $g(t, 0, y)$ is known, so are its Fourier coefficients, $\left\{g_{k}(t)\right\}_{k \geq 1}$

$$
g(t, 0, y)=\sum_{k \geq 1} g_{k}(t) s_{k} \sin \left(y v_{k}\right)
$$


and so we have by (4.14), 4.15), and (4.12) the integral equation in $\rho_{k}$

$$
g_{k}(t)=\sum_{n \geq 1} c_{n k}(t) r_{n}=\sum_{n \geq 1} r_{n} d_{n k}(t)+\int_{0}^{t} \rho_{k}(\eta) \sum_{n \geq 1} r_{n}^{2} a_{n} e^{-\lambda_{n k}(t-\eta)} d \eta .
$$

Thus the problem reduces to finding $\left\{\rho_{k}\right\}$ by solving the Volterra equation of the first kind 4.16 , where $\delta_{k}(t)=g_{k}(t)-$ $\sum_{n \geq 1} r_{n} d_{n k}(t)$ and $\mathcal{E}_{k}(t)=\sum_{n \geq 1} r_{n}^{2} a_{n} e^{-\lambda_{n k} t}$ are given.

Thus we can rewrite the integral equation (4.16) as a Volterra convolution equation of the first kind,

$$
\int_{0}^{t} \mathcal{E}_{k}(t-\eta) \rho_{k}(\eta) d \eta=\delta_{k}(t)
$$

Since $\mathcal{E}_{k}(0)=\sum_{n \geq 1} r_{n}^{2} a_{n}=1$, it is easily transformed into second kind by differentiating it

$$
\rho_{k}(t)+\int_{0}^{t} \mathcal{E}_{k}^{\prime}(t-\eta) \rho_{k}(\eta) d \eta=\delta_{k}^{\prime}(t)
$$

where

$$
\delta_{k}^{\prime}(t)=g_{k}^{\prime}(t)-\sum_{n \geq 1} r_{n} d_{n k}^{\prime}(t) \quad \text { and } \quad \mathcal{E}_{k}^{\prime}(t)=-\sum_{n \geq 1} r_{n}^{2} a_{n} \lambda_{n k} e^{-\lambda_{n k} t}
$$

are given continuous functions. Thus by using successive approximations we can easily solve the Volterra equation, 4.17, and recover the solution $\rho_{k}$ over any interval $(0, T)$. Most importantly the solution can be expressed through an integral operator whose kernel is the resolvent $\boldsymbol{R}_{k}$

$$
\rho_{k}(t)=\delta_{k}^{\prime}(t)+\int_{0}^{t} R_{k}(t-\eta) \delta_{k}^{\prime}(\eta) d \eta \quad \text { for } \quad t>0 .
$$

Recall that although the resolvent kernel $R_{k}$ can be obtained by iterating the kernel $\mathcal{E}_{k}^{\prime}$, it is also the solution of the Volterra integral equation

$$
-\mathcal{E}_{k}^{\prime}(t)=R_{k}(t)+\int_{0}^{t} \mathcal{E}_{k}^{\prime}(t-\eta) R_{k}(\eta) d \eta \quad \text { for } \quad t>0 .
$$

Since (4.20) is also a convolution equation, the Laplace transform can be used to find $R_{k}$. As we already have

$$
\mathcal{E}_{k}^{\prime}(t)=O\left(e^{-\lambda_{1 k} t}\right),
$$

let us denote by

$$
m=\sup _{t \geq 0}\left|\mathcal{E}_{k}^{\prime}(t)\right|
$$

The solution $R_{k}$ exists and is continuous as it is obtained by successive iterations, and it is also known that it grows at most exponentially, see 24 I-53

$$
R_{k}(t)=O\left(e^{m t}\right)
$$

Thus its Laplace transform, $\mathcal{L}\left(R_{k}\right)(s)$, is well defined and analytic for $\operatorname{Re}(s)>m$, and satisfies, 4.20,

$$
\mathcal{L}\left(R_{k}\right)(s)+\mathcal{L}\left(\mathcal{E}_{k}^{\prime}\right)(s)+\mathcal{L}\left(\mathcal{E}_{k}^{\prime}\right)(s) \mathcal{L}\left(R_{k}\right)(s)=0 \quad \text { for } \quad \operatorname{Re}(s)>\max \left\{m, \lambda_{1 k}\right\}
$$

or

$$
\mathcal{L}\left(R_{k}\right)(s)=\frac{-\mathcal{L}\left(\mathcal{E}_{k}^{\prime}\right)(s)}{1+\mathcal{L}\left(\mathcal{E}_{k}^{\prime}\right)(s)} \quad \text { for } \quad \operatorname{Re}(s)>\max \left\{m, \lambda_{1 k}\right\} .
$$

Therefore we can recover $R_{k}$ by taking the inverse Laplace transform

$$
R_{k}(t)=\mathcal{L}^{-1}\left(\frac{-\mathcal{L}\left(\mathcal{E}_{k}^{\prime}\right)(s)}{1+\mathcal{L}\left(\mathcal{E}_{k}^{\prime}\right)(s)}\right)(t)
$$

where from (4.20) and (4.21) the denominator do not vanish on $\left\{s \in \mathbb{C} ; \operatorname{Re}(s)>\max \left\{m, \lambda_{1 k}\right\}\right\}$. Then we can use (4.19) to find each $\rho_{k}$ over any interval $(0, T)$. Summing them up will help reconstruct $F_{1}$.

This concludes the computation of $p\left(t, \ell_{1}, y\right)$ which is the temperature of the membrane. 


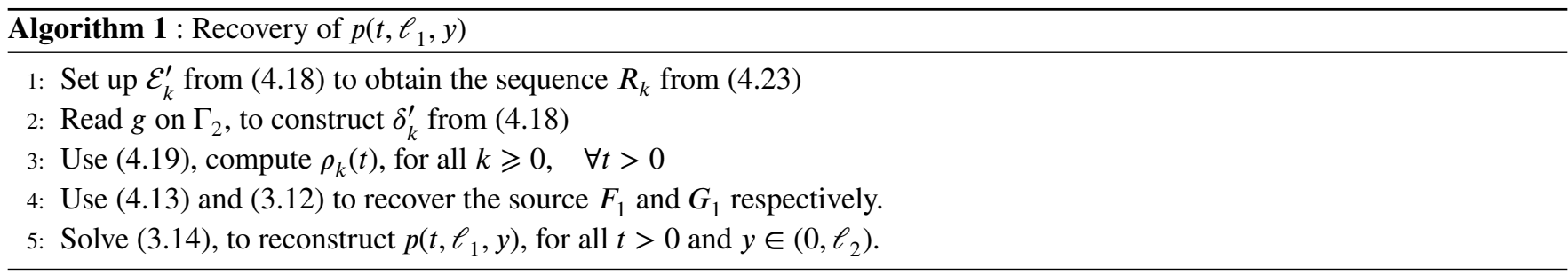

Remark 1. Equation (4.19) is an essential step of the algorithm as it allows to feed the reading $g_{k}$ and obtain $\rho_{k}$ in real time, and so makes the monitoring of the temperature of the membrane $p\left(t, \ell_{1}, y\right)$ also in real time possible. We can further monitor the temperature in the whole domain $\Omega$, if needs be, since we now have the coefficients $c_{n k}(t)$ from (4.12). Also anomalies in the temperature distribution and hot spots found on the membrane allow for an early detection of possible rupture of the membrane and so helps prevent costly shutdowns.

\section{ACKNOWLEDGMENT}

Research reported in this publication has been supported by the King Abdullah University of Science and Technology (KAUST), Saudi Arabia.

\section{CONFLICT OF INTERESTS}

This work does not have any conflicts of interest.

\section{References}

1. Khayet M. Membrane distillation (Chap. 6), in: N N Li, AG Fane, WSW Ho, T Matsuura (Eds.), Advanced Membrane Technology and Applications. John Wiley \& Sons, Inc.; 2008.

2. Ghaffour N, Bundschuh J, Mahmoudi H, Goosen M F. Renewable energy-driven desalination technologies: A comprehensive review on challenges and potential applications of integrated systems. Desalination. 2015;356:94-114.

3. Eleiwi F, Laleg-Kirati T M. Observer-based perturbation extremum seeking control with input constraints for direct-contact membrane distillation process. International Journal of Control. 2018;91(6):1363-1375.

4. Wang G-S., Ke H, Gray S, Moon I-S. Solar energy assisted direct contact membrane distillation (DCMD) process for seawater desalination. Separation and Purification Technology. 2015;143:94-104.

5. Naidu G, Shim W G, Jeong S, Choi Y, Ghaffour N, Vigneswaran S. Transport phenomena and fouling in vacuum enhanced direct contact membrane distillation: Experimental and modelling. Separation and Purification Technology. 2017;172:285295.

6. Eleiwi F, Ghaffour N, Alsaadi A S, Francis L, Laleg-Kirati T M. Dynamic modeling and experimental validation for direct contact membrane distillation (DCMD) process. Desalination. 2016;384:1-11.

7. Ghattassi M, Laleg T-M, Vivalda J-C. Analysis and Output Tracking Design for the Direct Contact Membrane distillation system. Preprint,https://arxiv.org/abs/1902.00964

8. Kimura T, Suzuki T. A parabolic inverse problem arising in a mathematical model for chromatography. SIAM Journal on Applied Mathematics. 1993;53(6):1747-1761.

9. Martin J, Wilcox L C, Burstedde C, Ghattas O. A stochastic Newton MCMC method for large-scale statistical inverse problems with application to seismic inversion. SIAM Journal on Scientific Computing. 2012;34(3):1460-1487. 
10. Kaltenbacher B, Klibanov M V. An inverse problem for a nonlinear parabolic equation with applications in population dynamics and magnetics. SIAM Journal on Mathematical Analysis. 2008;39(6):1863-1889.

11. Ammari H, Chow Y T, Zou J. Phased and phaseless domain reconstructions in the inverse scattering problem via scattering coefficients. SIAM Journal on Applied Mathematics. 2016;76(3):1000-1030.

12. Ammari H, Garnier J, Jugnon V, Kang H. Direct Reconstruction Methods in Ultrasound Imaging of Small Anomalies (Lecture Notes in Mathematics vol 2035). 2011.

13. Al-Musallam F, Boumenir A. Reconstruction of the Refraction Index in Stratified Ocean. SIAM J. Appl. Math.. 2011;71:972982.

14. Boumenir A, Tuan V K. Recovery of the heat equation from a single boundary measurement. Applicable Analysis. 2017;:110.

15. Boumenir A. The reconstruction of a parabolic system. Mathematical Methods in the Applied Sciences. 2017;40(16):58815892.

16. Avdonin S, Bell J, Nurtazina K. Determining distributed parameters in a neuronal cable model on a tree graph. Mathematical Methods in the Applied Sciences. 2017;40(11):3973-3981.

17. Avdonin S, Bell J. Determining a distributed conductance parameter for a neuronal cable model defined on a tree graph. Journal of Inverse Problems and Imaging. 2015;9(3):645-659.

18. Al-Musallam F, Boumenir A. The reconstruction of a source and a potential from boundary measurements. Journal of Mathematical Analysis and Applications. 2016;435(1):800-808.

19. Ammari K, Choulli M. Logarithmic stability in determining two coefficients in a dissipative wave equation. Extensions to clamped Euler-Bernoulli beam and heat equations. Journal of Differential Equations. 2015;259(7):3344-3365.

20. Ammari K, Choulli M, Triki F. Determining the potential in a wave equation without a geometric condition. Extension to the heat equation. Proceedings of the American Mathematical Society. 2016;144(10):4381-4392.

21. Imanuvilov O Y, Yamamoto M. Lipschitz stability in inverse parabolic problems by the Carleman estimate. Inverse problems. 1998;14(5):1229.

22. Choulli M, Yamamoto M. Global existence and stability for an inverse coefficient problem for a semilinear parabolic equation. Archiv der Mathematik. 2011;97(6):587-597.

23. Ammari K, Choulli M, Triki F. Hölder stability in determining the potential and the damping coefficient in a wave equation. Journal of Evolution Equations. 2019;19(2):305-319.

24. Smirnov V. Cours de mathématiques supérieures. Tome IV, 1 partie, edition Mir; 1975. 